\title{
Clinical implication of Tiam1 overexpression in the prognosis of patients with serous ovarian carcinoma
}

\author{
HUIWEN LI ${ }^{1,2^{*}}$, XUELIAN CUI $^{1 *}$, DINGBAO CHEN ${ }^{3}$, YANG YANG $^{1}$, JUNJIE PIAO $^{1}$, \\ ZHENHUA LIN ${ }^{1}$, GUANGHAI YAN ${ }^{1}$ and DANHUA SHEN ${ }^{3}$ \\ ${ }^{1}$ Cancer Research Center, Yanbian University, Yanji, Jilin 133002; ${ }^{2}$ Department of Pediatrics, Yanbian University Hospital, \\ Yanji, Jilin 133000; ${ }^{3}$ Department of Pathology, The People's Hospital of Beijing University, Beijing 100044, P.R. China
}

Received May 29, 2015; Accepted August 9, 2016

DOI: $10.3892 / \mathrm{ol} .2016 .5091$

\begin{abstract}
T lymphoma invasion and metastasis 1 (Tiaml), a guanine nucleotide exchange factor, was originally identified as an invasion- and metastasis-inducing gene in T lymphoma cells. High expression levels of the human Tiaml gene have been found in numerous human malignancies, suggesting a potential role as a modifier of tumor initiation and progression. However, little is known about the status of Tiam 1 in ovarian carcinoma. The present study aimed to investigate the clinicopathological significance of high Tiaml expression in serous ovarian carcinoma. Immunohistochemical staining for Tiam1 was performed in 182 patients with serous ovarian carcinoma, in 76 patients with ovarian borderline tumors and in 72 patients with benign ovarian tumors. Immunofluorescence staining was also performed to detect the subcellular localization of Tiam1 protein in SK-OV-3 ovarian carcinoma cells. The correlations between high Tiam1 expression and the clinicopathological features of the ovarian carcinomas were evaluated by the $\chi^{2}$ test and Fisher's exact test. The overall survival (OS) rates were calculated by the Kaplan-Meier method, and the association between prognostic factors and patient survival was analyzed by the Cox proportional hazard model. Tiam1 protein showed a cytoplasmic and nuclear staining pattern in ovarian carcinoma. Strongly-positive Tiam1 protein expression was observed in 59.3\% (108/182) of ovarian carcinomas, which was significantly higher than in benign serous tumors $(12.5 \%$; 9/72). Moreover, the rate
\end{abstract}

Correspondence to: Professor Danhua Shen, Department of Pathology, The People's Hospital of Beijing University, 11 South Avenue, Xi Zhi Men Xicheng, Beijing 100044, P.R. China

E-mail: shenpath59@163.com

Professor Guanghai Yan, Cancer Research Center, Yanbian University, 977 Gongyuan Street, Yanji, Jilin 133002, P.R. China

E-mail: ghyan@ybu.edu.cn

*Contributed equally

Key words: ovarian carcinoma, Tiam1, immunohistochemistry, prognosis, survival analysis of strongly-positive Tiam1 expression in borderline serous tumors $(31.6 \% ; 24 / 76)$ was also significantly higher than that in benign serous tumors. High Tiam1 protein expression was closely associated with a high histological grade, metastasis, advanced clinical stage and lower OS rates in ovarian carcinoma. Multivariate analysis indicated that Tiam1 was an independent prognostic factor, along with metastasis and clinical stage, in patients with ovarian carcinoma. In conclusion, Tiam1 expression is strongly associated with grade and outcome in ovarian carcinoma, and may serve as a useful molecular marker for clinical management.

\section{Introduction}

Detecting carcinoma at an early stage or tackling it at the late stage in an efficient way is always a challenge in clinical oncology. As one of the most frequently occurring gynecological malignancies, ovarian carcinoma is the fifth most common cause of cancer-associated mortality in women (1). The poor clinical outcome is likely to be caused, at least in part, by the high percentage of cases diagnosed at an advanced stage. Tumor recurrence and chemoresistance are common reasons for carcinoma-related mortality (2). Understanding the possible mechanisms underlying the aggressive progression of ovarian carcinoma may, therefore, provide better prognostic and predictive factors for this disease.

T lymphoma invasion and metastasis protein (Tiam1) was first identified as a gene that induces invasion and metastasis using $\mathrm{T}$ lymphoma cells by proviral tagging and in vitro selection for invasiveness $(3,4)$. Tiam1 is a guanine nucleotide exchange factor that mediates the specific activation of Rac1 (5-7). Tiam1 maintains the specificity of Rac1 toward specific downstream effector pathways, whereas Racl regulates cell survival and cell cycle progression $(8,9)$. Together, Tiam1-Rac1 is a critical component in the biology of human tumors, affecting the transformed cells themselves and the tumor microenvironment (10-12). Tiam1 is a potent modifier of oncogenic Ras-induced skin tumor initiation, promotion and progression (13). Moreover, numerous studies have demonstrated that the upregulation of Tiam1 is associated with an aggressive phenotype and a poor clinical outcome in several types of malignant tumors, including breast (14), colon (15), prostate (16), liver (17) and nasopharyngeal (11) tumors, and 
esophageal squamous cell carcinoma (18). However, to date, the correlation between Tiam1 expression and ovarian carcinoma has not been adequately investigated.

The current study was undertaken to investigate the expression of Tiam1 in human ovarian benign, borderline and malignant tumors, and to investigate whether Tiam1 expression is associated with the clinicopathological features of ovarian carcinoma. Furthermore, the prognostic value of high Tiam 1 expression in patients with serous ovarian carcinoma was also assessed.

\section{Materials and methods}

Ethics statement. This study complied with the Declaration of Helsinki and was approved by the Human Ethics and Research Ethics committees of Yanbian University Medical College (Yanji, Jilin, China) and The People's Hospital of Beijing University (Beijing, China). Through the use of surgical consent forms, patients were informed that the resected specimens would be stored by the hospital and potentially used for scientific research, and that their privacy would be maintained. Follow-up survival data were collected retrospectively through medical record analyses.

Patients and tissue specimens. A total of 330 human ovarian tumor specimens, including 182 serous carcinomas, 76 borderline serous tumors and 72 benign serous tumors, were used for this study. These tumors were selected randomly from female patients undergoing surgery between February 2006 and October 2010, and stored in the Tumor Tissue Bank of Yanbian University Medical College. Pathological parameters, including age, menopausal status, grade, metastasis and survival data, were carefully reviewed in all 182 serous ovarian carcinomas.

In these 182 cases, the patients ages ranged from 16 to 75 years, with a mean age of 48.3 years, and the patient age ratio between $\geq 48$ years to $<48$ years was 101:81. All samples were routinely fixed in $10 \%$ buffered formalin and embedded in paraffin, and tissue sections $(4-\mu \mathrm{m})$ were stained with hematoxylin and eosin $(\mathrm{H} \& \mathrm{E})$. The $\mathrm{H} \& \mathrm{E}$-stained slides of the different biopsies were reviewed by two experienced pathologists and one appropriate paraffin block was selected for this study. Histopathological grading was performed using the World Health Organization (Pathology and Genetics Tumors of Gynecological System) criteria (with 72 low-grade tumors and 110 high-grade tumors) (19). All the ovarian carcinoma patients were clinically staged according to the International Federation of Gynecology and Obstetrics (FIGO) staging system [with 86 early-stage tumors (FIGO stages I and II) and 96 late-stage tumors (FIGO stages III and IV)] (19). None of the ovarian carcinoma patients received pre-operative radiation or chemotherapy prior to surgery. All ovarian carcinoma patients had follow-up records of longer than 5 years.

Immunohistochemical (IHC) analysis. IHC analysis was performed using the DAKO LSAB kit (DAKO A/S, Glostrup, Denmark). Briefly, to eliminate endogenous peroxidase activity, $4-\mu \mathrm{m}$ thick tissue sections were deparaffinized, rehydrated and incubated with $3 \% \mathrm{H}_{2} \mathrm{O}_{2}$ in methanol for $15 \mathrm{~min}$ at room temperature (RT). The antigen was retrieved at $95^{\circ} \mathrm{C}$ for
$20 \mathrm{~min}$ by placing the slides in $0.01 \mathrm{M}$ sodium citrate buffer (pH 6.0). The slides were then incubated with the polyclonal anti-C-terminal Tiam1 antibody (1:100 dilution; catalog no. SC-872; Santa Cruz Biotechnology Inc., Dallas, TX, USA) at $4^{\circ} \mathrm{C}$ overnight. Following incubation with the biotinylated secondary antibody (catalog no. PV9000; ZSGB-Bio, Beijing, China) at RT for $30 \mathrm{~min}$, the slides were incubated with streptavidin-peroxidase complex at RT for $30 \mathrm{~min}$. Immunostaining was developed using 3,3'-diaminobenzidine, and Mayer's hematoxylin was used for counterstaining (20). Rabbit immunoglobulin G (IgG) (1:2,500 dilution; catalog no. GTX35035; GeneTex Inc., Irvine, CA, USA) was used as an isotope control. In addition, positive tissue sections were processed while omitting the primary antibody (rabbit anti-Tiam1) for negative controls.

As described previously (21), expression was scored by two pathologists who did not possess knowledge of the clinical data. In case of discrepancies, a final score was established by reassessment on a double-headed microscope. Briefly, the immunostaining for Tiam1 was semi-quantitatively scored as follows: -, no or $<5 \%$ positive cells;,$+ 5-25 \%$ positive cells; ,$++ 26-50 \%$ positive cells; and,$+++>50 \%$ positive cells). The cytoplasmic and nuclear expression patterns were each considered as a positive staining result. Tissue sections scored as ++ and +++ were considered as strong positive results (high expression) for Tiam1. For the survival data analysis, ++ or +++ samples were considered as high Tiam1 expression and - or + samples were considered as low Tiam1 expression.

Immunofluorescence (IF) staining analysis. IF staining was used to detect the subcellular localization of Tiam1 protein in ovarian carcinoma SK-OV-3 cells (Korean Cell Line Bank, Seoul, Korea). All steps were performed at RT. SK-OV-3 cells were grown to $70-80 \%$ confluence on coverslips, then fixed with $4 \%$ paraformaldehyde for $10 \mathrm{~min}$ and permeabilized with $0.5 \%$ Triton X-100 for 10 min after $24 \mathrm{~h}$. Subsequent to being blocked with 3\% Albumin Bovine V (catalog no. A8020; Solarbio, Beijing, China) for $1 \mathrm{~h}$, the slides were quickly and gently washed with phosphate-buffered saline (PBS). The cells were then incubated with the Tiam1 antibody (1:100 dilution) at $4^{\circ} \mathrm{C}$ overnight, followed by incubation with Alexa Fluor ${ }^{\circledR} 488$ goat anti-rabbit $\operatorname{IgG}(\mathrm{H}+\mathrm{L})$ (catalog no. A11004; 1:1,000 dilution; Invitrogen; Thermo Fisher Scientific, Inc., Waltham, MA, USA) for $1 \mathrm{~h}$. After washing with PBS, the cells were counterstained with 4',6-diamidino-2-phenylindole (catalog no. C1006; Beyotime, Shanghai, China) and the coverslips were mounted with Antifade Mounting Medium (catalog no. P0126; Beyotime) (21). Finally, the IF signals were visualized and recorded using a Leica SP5II confocal microscope (Leica Microsystems GmbH, Wetzlar, Germany).

Statistical analysis. Statistical analyses included descriptive statistics with determination of minimal and maximal values, means and medians, with $95 \%$ confidence intervals (CIs) for particular variables. The $\chi^{2}$ test and Fisher's exact test were used to assess correlations between clinicopathological characteristics and the expression of the studied protein. The Kaplan-Meier method was used to calculate the survival rates after tumor removal and the Log-rank test was used to analyze the differences in survival curves. Multivariate survival 

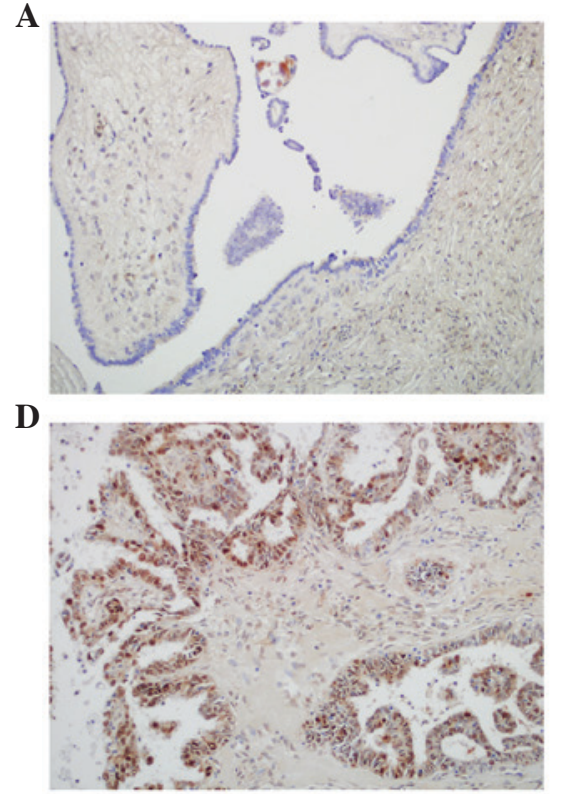

B

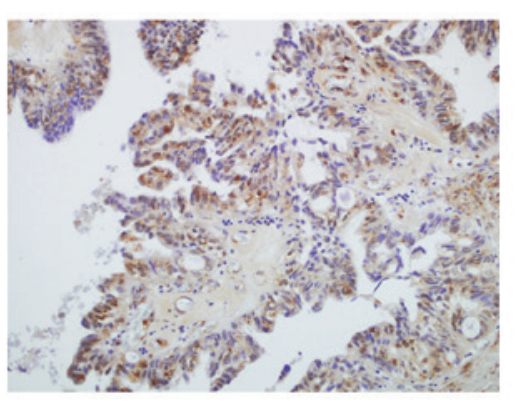

$\mathbf{E}$

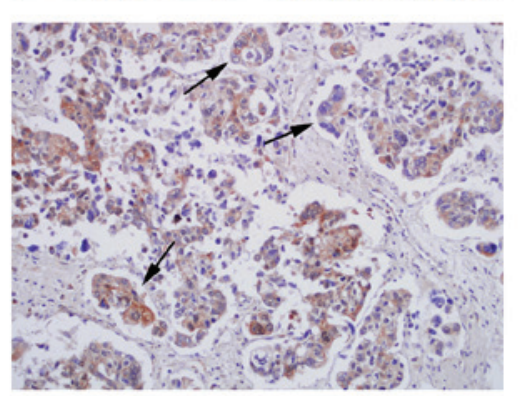

C

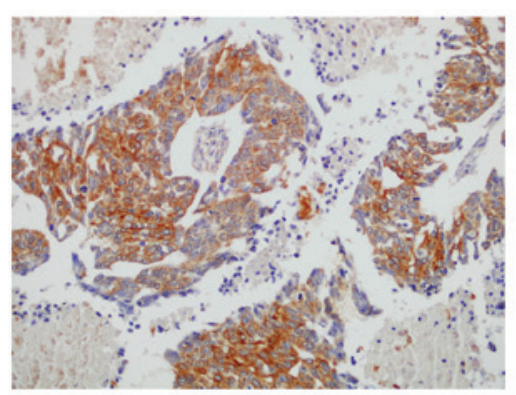

F

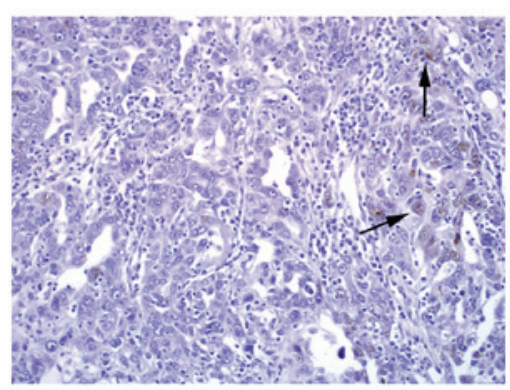

Figure 1. Immunohistochemical staining of T lymphoma invasion and metastasis 1 (Tiam1) protein in ovarian tumor samples. (A) Negative expression of Tiam1 protein in a benign serous tumor. (B) Positive expression of Tiam1 protein in a borderline serous tumor. (C) Strong positive expression of Tiam1 protein in serous carcinoma cells, showing a cytoplasmic staining pattern. (D) A mainly nuclear staining pattern in serous carcinoma cells. (E) Positive expression of Tiam1 protein in the metastatic serous carcinoma cells in the vascular/lymphatic vessels (arrows). (F) Negative or weakly-positive expression of Tiam1 protein in serous carcinoma without metastasis. Original magnification, x200.

analysis was performed on all the characteristics measured by univariate survival analysis (age, menopausal status, histological grade, metastasis, FIGO stage and Tiam1 expression) through the Cox proportional hazard regression model. Statistical analyses were performed using the SPSS software program for windows, version 17.0 (SPSS, Inc, Chicago, IL, USA), and the JMP software program for Mac, version 10.0.0 (SAS Institute Inc, Cary, NC, USA). $\mathrm{P}<0.05$ was considered to indicate a statistically significant difference.

\section{Results}

Tiaml protein is overexpressed in serous ovarian carcinoma. IHC staining for the Tiam1 protein was performed in 182 ovarian carcinoma tissues. The analysis of Tiam1 expression in the ovarian carcinoma cells from the 182 patients revealed predominantly cytoplasmic and nuclear expression patterns in the positive cases (Fig. 1). The IF staining results also showed that Tiam 1 protein was localized in the cytoplasm and nuclei of the ovarian carcinoma SK-OV-3 cells (Fig. 2).

IHC staining indicated that the rate of positive Tiam1 protein expression was significantly higher in serous carcinomas $(75.8 \% ; 138 / 182)$ than in benign serous tumors $(36.1 \%$; 26/72) $(\mathrm{P}<0.01)$. Similarly, the rate of strongly positive Tiam1 protein expression was significantly higher in serous carcinomas $(59.3 \%$; 108/182) than in benign serous tumors $(12.5 \%$; $9 / 72)(\mathrm{P}<0.01)$. Moreover, the rates of positive $(51.3 \%$; $39 / 76)$ and strongly-positive $(31.6 \% ; 24 / 76)$ Tiam1 protein expression in borderline serous tumors were significantly higher than those in benign serous tumors $(\mathrm{P}<0.05$ and $\mathrm{P}<0.01$, respectively) (Table I).

Correlations between Tiaml expression status and clinicopathological features of serous ovarian carcinoma. To

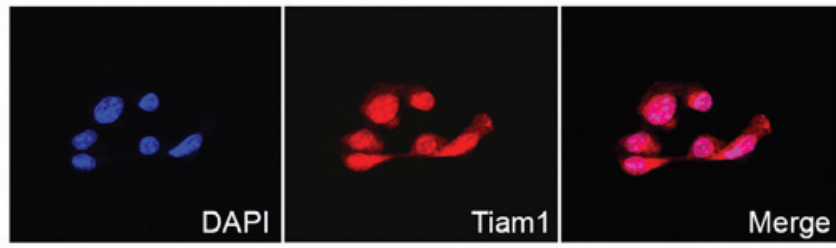

Figure 2. Immunofluorescence staining of the T lymphoma invasion and metastasis 1 (Tiam1) protein in ovarian carcinoma SK-OV-3 cells. The Tiam 1 protein was localized in the cytoplasm and the nucleus of the cells [red indicates Tiam1 staining, blue indicates 4',6-diamidino-2-phenylindole (DAPI) staining].

evaluate the association between Tiam1 protein and ovarian carcinoma progression, the correlations between high Tiam1 expression and the clinicopathological features of ovarian carcinomas were analyzed. The rate of strongly-positive Tiam1 protein expression was significantly higher in high-grade $(66.4 \% ; 73 / 110)$ ovarian carcinomas than in low-grade cases $(48.6 \% ; 35 / 72)(\mathrm{P}=0.017)$, and in ovarian carcinomas with metastasis $(73.2 \%$; 71/97) than in cases without metastasis $(43.5 \%$; 37/85) $(\mathrm{P}<0.001)$. Regarding FIGO clinical stages, the rate of strongly-positive Tiam1 expression was $69.8 \%(67 / 96)$ in the late-stage (IIB-IIIC) ovarian carcinomas, but only $47.7 \%$ $(41 / 86)$ in the early-stage (I-IIA) cases $(\mathrm{P}=0.002)$. However, high expression of Tiam1 protein was not associated with the age or menopausal status of the patients with ovarian carcinoma (Fig. 3; Table II).

High Tiaml expression is an independent biomarker of a poor prognosis in patients with serous ovarian carcinoma. To further substantiate the importance of high Tiam1 expression in ovarian carcinoma progression, the overall survival (OS) rate of 182 ovarian carcinoma patients was assessed 
Table I. T lymphoma invasion and metastasis 1 protein expression in ovarian carcinoma.

\begin{tabular}{lrrrrrrr}
\hline & & \multicolumn{3}{c}{ Positive cases, $\mathrm{n}$} & & \\
\cline { 3 - 5 } Diagnosis & No. of cases & - & + & ++ & +++ & Positive cases, \% & Strongly-positive cases, \% \\
\hline Serous carcinoma & 182 & 44 & 30 & 72 & 36 & $75.8^{\mathrm{a}}$ & $59.3^{\mathrm{a}}$ \\
Borderline serous tumor & 76 & 37 & 15 & 19 & 5 & $51.3^{\mathrm{b}}$ & $31.6^{\mathrm{a}}$ \\
Benign serous tumor & 72 & 46 & 17 & 9 & 0 & 36.1 & 12.5 \\
\hline
\end{tabular}

${ }^{a} \mathrm{P}<0.01$ and ${ }^{\mathrm{b}} \mathrm{P}<0.05$ compared with benign serous tumor.
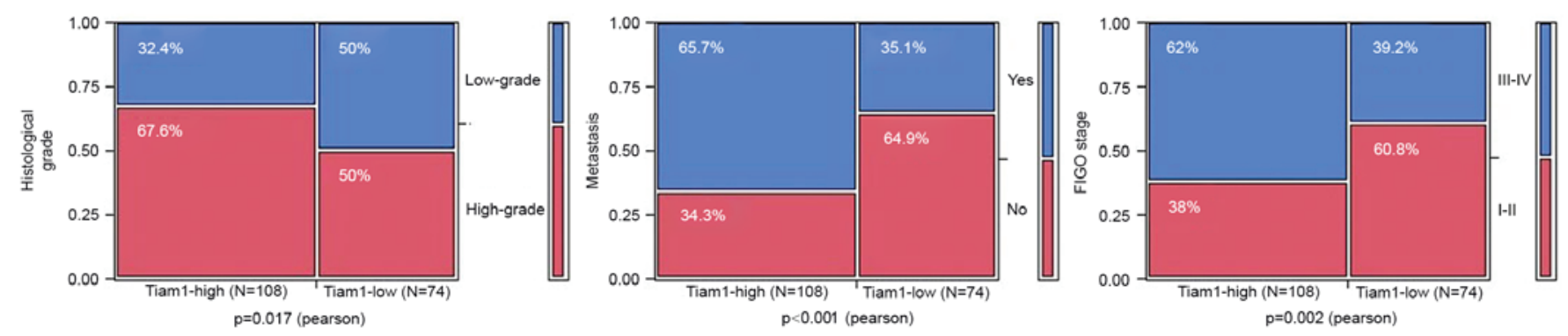

Figure 3. Correlations between T lymphoma invasion and metastasis 1 (Tiam1) expression and the clinicopathological significance of ovarian carcinoma. The expression level of Tiam 1 protein was significantly associated with histological grade $(\mathrm{P}=0.017)$, metastasis $(\mathrm{P}<0.001)$ and International Federation of Gynecology and Obstetrics (FIGO) stage ( $\mathrm{P}=0.002)$.

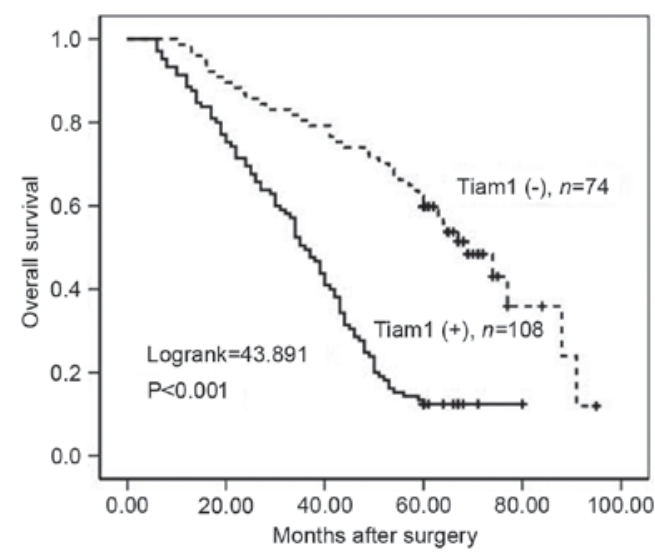

Figure 4. Kaplan-Meier survival curves illustrating the significance of T lymphoma invasion and metastasis 1 (Tiam1) expression in ovarian carcinomas. Overall survival rates of patients with high and low Tiam1 expression.

using the Kaplan-Meier method. Patients with high Tiam1 expression exhibited a lower OS rate than those with low Tiam1 expression (Log-rank=43.891, P<0.001) (Fig. 4). Similarly, ovarian carcinoma patients with high Tiam1 expression exhibited decreased OS rates compared with those with low Tiam1 expression in early-stage cases (Log-rank $=14.430$, $\mathrm{P}<0.001)$ and late-stage cases $(\log -\mathrm{rank}=23.562, \mathrm{P}<0.001)$ (Fig. 5). Notably, high Tiam1 expression was associated with significantly lower OS rates in cases with metastasis $($ Log-rank=15.087, $\mathrm{P}<0.001)$ and without metastasis (Log-rank=17.337, $\mathrm{P}<0.001$ ) (Fig. 6). Moreover, the survival rate of patients with low-grade (Log-rank=19.293, $\mathrm{P}<0.001)$ and high-grade $(\mathrm{Log}-\mathrm{rank}=21.038, \mathrm{P}<0.001)$ ovarian carcinoma was significantly lower in patients with tumors exhibiting high Tiam1 expression compared with those exhibiting low expression (Fig. 7).

Univariate analysis demonstrated that histological grade $(\mathrm{P}=0.019)$, metastasis $(\mathrm{P}<0.001)$, FIGO stage $(\mathrm{P}<0.001)$ and Tiam1 expression status $(\mathrm{P}<0.001)$ were all significantly associated with OS rate in patients with ovarian carcinoma. These data suggested that Tiam 1 may be a valuable prognostic factor in ovarian carcinoma. Multivariate analysis was subsequently performed using the Cox proportional hazards model for all variables examined in the univariate analysis. It was found that the high expression of Tiam1 (HR, 2.559; 95\% CI, 1.788-3.663; P<0.001), FIGO stage (HR, 2.530; 95\% CI, 1.835-3.488; $\mathrm{P}<0.001)$ and metastasis $(\mathrm{HR}, 2.088$; 95\% CI, 1.493-2.919; $\mathrm{P}<0.001)$ were significant independent prognostic factors for survival in ovarian carcinoma (Table III).

\section{Discussion}

Tiam1 exhibits a number of roles in the regulation of cellular functions depending on the specific cell type, substratum and other factors (17). As a guanine nucleotide exchange factor, Tiam1 has been shown to be essential in a range of tumor signaling pathways through the regulation of Rho GTPase functions (22). It has been shown that Tiam1 is involved in the rearrangement of the cytoskeleton, the migration of cells, and the mobility of T-lymphoma cells, fibroblasts and epithelial cells $(5,6,23,24)$. Moreover, studies have indicated that Tiam1 is involved in anti- and pro-apoptotic mechanisms (25). Accordingly, alterations in Tiam1 expression/function may contribute to tumorigenesis and carcinoma progression of common types of human cancer. 
Table II. Correlations between Tiam1 protein expression and the clinicopathological parameters of ovarian carcinoma.

\begin{tabular}{|c|c|c|c|c|}
\hline Variables & No. of cases & Tiam1 strongly positive cases, n (\%) & $\chi^{2}$ & P-value \\
\hline Age, years & & & & 0.777 \\
\hline$\geq 48$ & 101 & $59(58.4)$ & 0.080 & \\
\hline$<48$ & 81 & $49(60.5)$ & & \\
\hline Menopausal status & & & & 0.371 \\
\hline Premenopausal & 86 & $54(62.8)$ & 0.804 & \\
\hline Postmenopausal & 96 & $54(56.3)$ & & \\
\hline Histological grade & & & & $0.017^{\mathrm{a}}$ \\
\hline Low-grade & 72 & $35(48.6)$ & 5.684 & \\
\hline High-grade & 110 & $73(66.4)$ & & \\
\hline Metastasis & & & & $<0.001^{\mathrm{b}}$ \\
\hline No & 85 & $37(43.5)$ & 16.525 & \\
\hline Yes & 97 & $71(73.2)$ & & \\
\hline FIGO stage & & & & $0.002^{\mathrm{b}}$ \\
\hline I-II & 86 & $41(47.7)$ & 9.197 & \\
\hline III-IV & 96 & $67(69.8)$ & & \\
\hline
\end{tabular}

${ }^{\mathrm{a}} \mathrm{P}<0.05$ and ${ }^{\mathrm{b}} \mathrm{P}<0.01$. Tiam1, T lymphoma invasion and metastasis 1; FIGO, International Federation of Gynecology and Obstetrics.

A

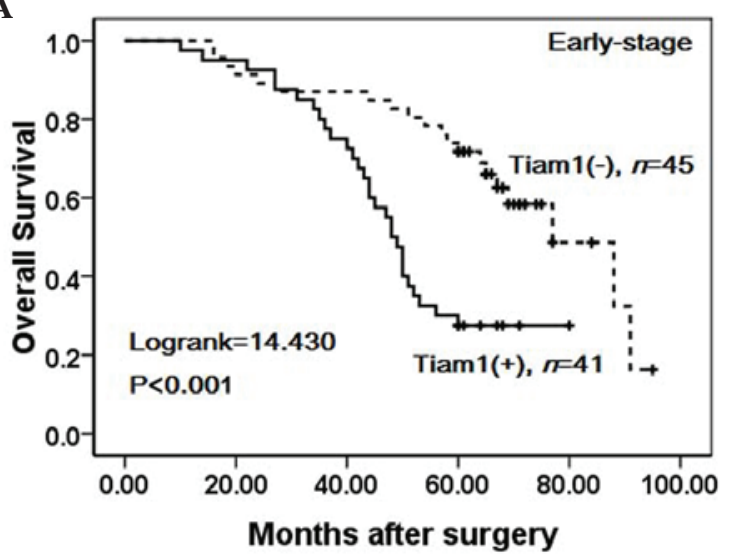

B

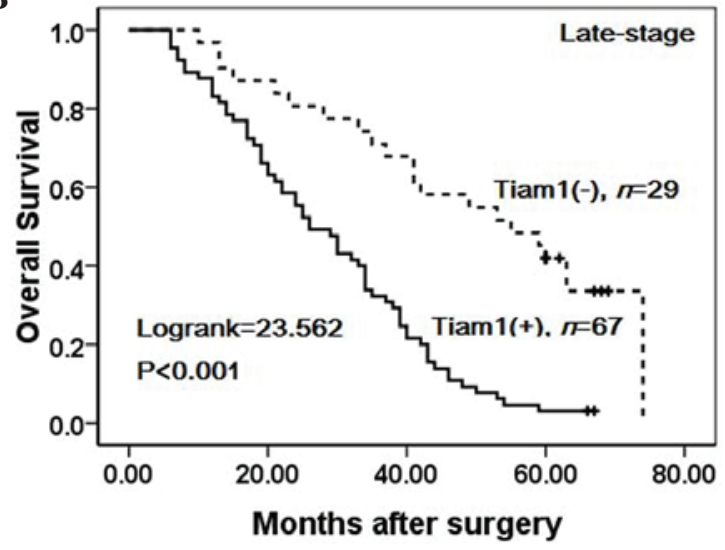

Figure 5. Kaplan-Meier survival curves illustrating the significance of T lymphoma invasion and metastasis 1 (Tiam1) expression in the early and late stages of ovarian carcinomas. High Tiam1 expression was strongly associated with the poor overall survival of patients with (A) early- or (B) late-stage ovarian carcinoma.

A

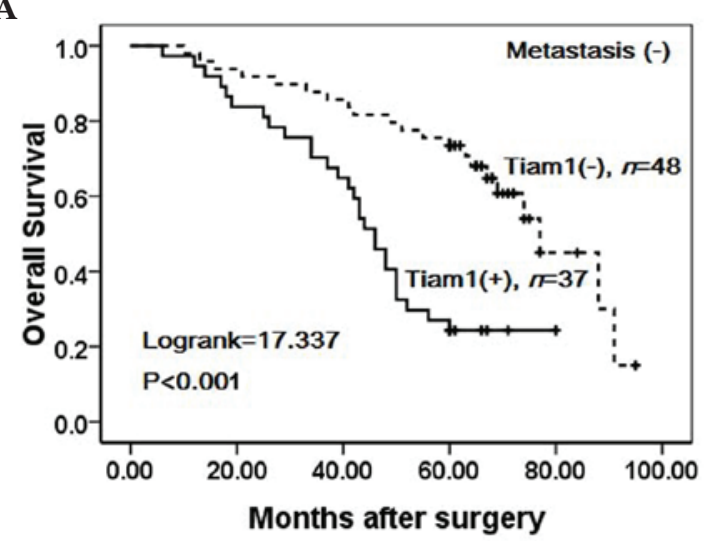

B

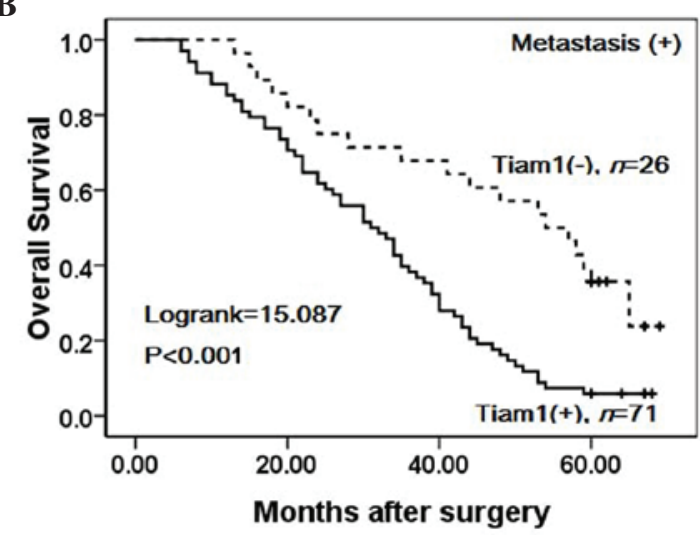

Figure 6. Kaplan-Meier survival curves illustrating the significance of T lymphoma invasion and metastasis 1 (Tiam1) expression in ovarian carcinomas with or without metastasis. High Tiam1 expression predicted poor overall survival in (A) patients without metastasis or (B) in those with metastasis of ovarian carcinoma. 
Table III. Univariate and multivariate analysis of clinicopathological factors for the overall survival rate of 182 patients with ovarian carcinoma.

\begin{tabular}{|c|c|c|c|c|}
\hline \multirow[b]{2}{*}{ Characteristics } & \multicolumn{2}{|c|}{ Univariate analysis } & \multicolumn{2}{|c|}{ Multivariate analysis } \\
\hline & HR $(95 \% \mathrm{CI})$ & P-value & $\mathrm{HR}(95 \% \mathrm{CI})$ & P-value \\
\hline Age, years & $1.079(0.802-1.453)$ & 0.615 & $0.979(0.715-1.340)$ & 0.894 \\
\hline Menopausal status & $1.093(0.811-1.472)$ & 0.559 & $0.920(0.660-1.283)$ & 0.624 \\
\hline Histological grade & $1.436(1.062-1.942)$ & $0.019^{\mathrm{a}}$ & $1.147(0.831-1.582)$ & 0.405 \\
\hline Metastasis & $2.739(1.981-3.787)$ & $<0.001^{\mathrm{b}}$ & $2.088(1.493-2.919)$ & $<0.001^{\mathrm{b}}$ \\
\hline FIGO stage & $2.705(1.978-3.699)$ & $<0.001^{\mathrm{b}}$ & $2.530(1.835-3.488)$ & $<0.001^{\mathrm{b}}$ \\
\hline Tiam1 & $2.854(2.075-3.927)$ & $<0.001^{b}$ & $2.559(1.788-3.663)$ & $<0.001^{\mathrm{b}}$ \\
\hline
\end{tabular}

${ }^{\mathrm{a}} \mathrm{P}<0.05$ and ${ }^{\mathrm{b}} \mathrm{P}<0.01$. HR, hazard ratio; $\mathrm{CI}$, confidence interval; Tiam1, T lymphoma invasion and metastasis 1; FIGO, International Federation of Gynecology and Obstetrics.

A

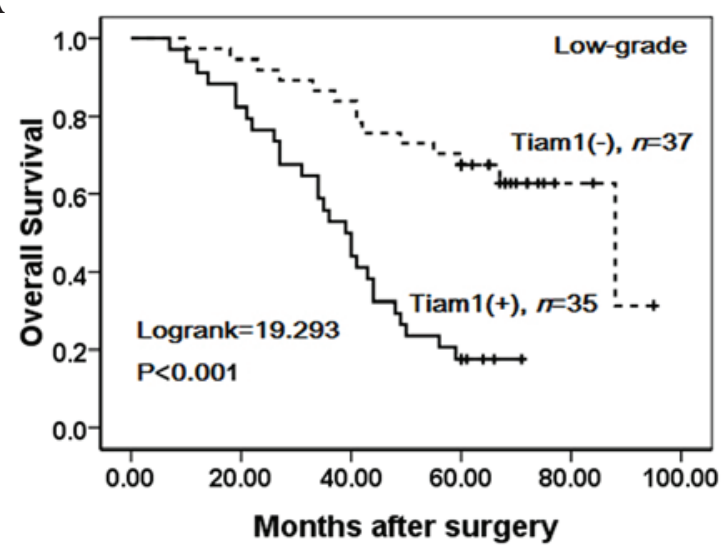

B

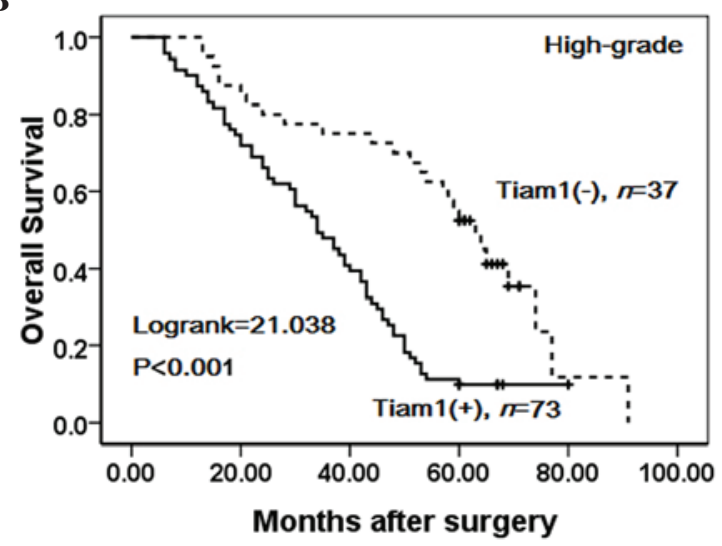

Figure 7. Kaplan-Meier survival curves illustrating the significance of T lymphoma invasion and metastasis 1 (Tiam1) expression in low- or high-grade ovarian carcinomas. High Tiam1 expression was strongly associated with poor overall survival in patients with (A) low- or (B) high-grade ovarian carcinoma.

Increasing evidence has focused on the regulation of Tiam1, as well as its role in carcinoma progression and metastasis. Liu et al found that Tiam1 expression was upregulated in lung carcinoma compared with normal lung tissues, and analysis further showed that Tiam1 overexpression was correlated with the lymph node metastasis of patients with lung carcinoma (22). Yu et al demonstrated that Tiam1 was upregulated in colorectal carcinoma tissues and provided evidence that Tiam1 was closely correlated to the metastatic potential of colorectal carcinoma. Depletion of Tiam1 significantly reduced the tumor growth and metastatic ability of colorectal carcinoma cells (26). However, its role in ovarian carcinoma remains to be elucidated. In the present study, IHC and IF staining of Tiam1 protein was performed in ovarian carcinoma tissues. It was found that positive staining of Tiam1 was mainly localized in the cytoplasm and nucleus. Compared with benign serous tumors, the positive and strongly-positive Tiam1 staining were significantly higher in serous carcinomas (both $\mathrm{P}<0.01$ ), which is consistent with the result of a previous study by Li et al (27), and it was also indicated that Tiam1 expression was markedly increased in primary and metastatic ovarian carcinoma tissues relative to normal ovarian tissues in a smaller group. These observations suggest that the high expression of Tiam1 may be correlated with the potential malignancy of ovarian carcinomas. Notably, a significant difference was also observed in the rates of positive and strongly-positive Tiam1 expression between borderline tumors and benign tumors $(\mathrm{P}<0.05$ and $\mathrm{P}<0.01)$, which may represent tumor progression. Nonetheless, studies have shown that borderline tumors and high-grade serous tumors have completely different etiologies rather than being high-grade tumors arising from borderline tumors (28). Accordingly, the present study did not compare the expression of Tiam1 in borderline tumors with that in serous carcinomas. Compatible with these findings, it was also observed that the strongly-positive Tiam1 protein expression rate was significantly higher in patients with late-stage serous ovarian carcinomas compared with that in patients with early-stage cancer. The analysis further showed that high Tiam1 expression correlates with the metastasis of patients with ovarian carcinoma, which suggests that Tiam1 may play an important role in the progression and invasion of ovarian carcinoma. Similarly, the strongly-positive Tiam1 protein expression rate was higher in patients with high-grade ovarian carcinoma compared with low-grade cases. High-grade serous ovarian carcinoma is the most lethal form of gynecological malignant carcinoma, and the majority of patients present with late clinical stages (FIGO stages III and 
IV) of disease at the time of diagnosis. These results demonstrate that the high expression of Tiam1 may assist in more accurate outcome prediction in serous ovarian carcinoma.

Despite the strong association between Tiam1 expression and cancer, reports of Tiam 1 expression-based outcome analysis in tumor patients are limited. Nevertheless, several reports have indicated that the high expression of Tiam1 is significantly associated with the shortened survival of patients with malignancies. For example, Engers et al reported that high Tiam1 expression is an independent predictor of decreased disease-free survival for patients with prostate cancer (16). Yang et al demonstrated that the high expression of Tiam1 correlates with a poor prognosis in hepatocellular carcinoma (29). Recently, Du et al suggested that high Tiam1 expression is associated with poor overall survival in patients with primary gallbladder carcinoma (10). With respect to survival, the present study found that ovarian carcinoma patients exhibiting high Tiam1 expression had lower OS rates compared with patients with low Tiam1 expression $(\mathrm{P}<0.01)$. Univariate survival analysis revealed that histological grade, metastasis, FIGO stage and Tiam1 expression status were all significantly associated with the OS of patients with serous ovarian carcinoma $(\mathrm{P}<0.05)$. Furthermore, multivariate survival analysis revealed that Tiam1 expression was an independent prognostic factor, as were metastasis and FIGO stage $(\mathrm{P}<0.01)$. These clinical and experimental data indicate that Tiam1 may be a useful prognostic factor and a potential therapeutic target in patients with serous ovarian carcinoma.

In conclusion, the high expression of Tiam1 appears to be significantly associated with ovarian carcinoma progression and is an independent prognostic factor, along with metastasis and clinical stage. Additional studies are warranted to further our understanding of the role that Tiam1 plays in ovarian carcinoma tumorigenesis.

\section{Acknowledgements}

This study was supported by grants from the Special Research Project of the 973 National Science and Technology Department of China (no. 2014CB560708) and the National Natural Science Funds of China (no. 81260665).

\section{References}

1. Siegel R, Ma J, Zou Z and Jemal A: Cancer statistics, 2014. CA Cancer J Clin 64: 9-29, 2014

2. Smolle E, Taucher V, Pichler M, Petru E, Lax S and Haybaeck J: Targeting signaling pathways in epithelial ovarian cancer. Int J Mol Sci 14: 9536-9555, 2013.

3. Habets GG, Scholtes EH, Zuydgeest D, van der Kammen RA, Stam JC, Berns A and Collard JG: Identification of an invasion-inducing gene, Tiam-1, that encodes a protein with homology to GDP-GTP exchangers for Rho-like proteins. Cell 77: 537-549, 1994.

4. Habets GG, van der Kammen RA, Stam JC, Michiels F and Collard JG: Sequence of the human invasion-inducing TIAM1 gene, its conservation in evolution and its expression in tumor cell lines of different tissue origin. Oncogene 10: 1371-1376, 1995.

5. Hordijk PL, ten Klooster JP, van der Kammen RA, Michiels F, Oomen LC and Collard JG: Inhibition of invasion of epithelial cells by Tiam1-Rac signaling. Science 278: 1464-1466, 1997.

6. Michiels F, Habets GG, Stam JC, van der Kammen RA and Collard JG: A role for Rac in Tiam1-induced membrane ruffling and invasion. Nature 375: 338-340, 1995.

7. Mertens AE, Roovers RC and Collard JG: Regulation of Tiam1-Rac signalling. FEBS Lett 546: 11-16, 2003.
8. Bollag G, Crompton AM, Peverly-Mitchell D, Habets GG and Symons M: Activation of Rac1 by human Tiam1. Methods Enzymol 325: 51-61, 2000.

9. Matsuo N, Terao M, Nabeshima Y and Hoshino M: Roles of STEF/Tiam1, guanine nucleotide exchange factors for Rac1, in regulation of growthcone morphology. Mol Cell Neurosci 24: 69-81, 2003.

10. Du X, Wang S, Lu J, Wang Q, Song N, Yang T, Dong R, Zang L, Yang Y, Wu T and Wang C: Clinical value of Tiam1-Rac1 signaling in primary gallbladder carcinoma. Med Oncol 29: 1873-1878, 2012

11. Qi Y, Huang B, Yu L, Wang Q, Lan G and Zhang Q: Prognostic value of Tiam1 and Rac1 overexpression in nasopharyngeal carcinoma. ORLJ Otorhinolaryngol Relat Spec 71: 163-171, 2009.

12. Xu K, Rajagopal S, Klebba I, Dong S, Ji Y, Liu J, Kuperwasser C, Garlick JA, Naber SP and Buchsbaum RJ: The role of fibroblast Tiam1 in tumor cell invasion and metastasis. Oncogene 29: 6533-6542, 2010

13. Malliri A, van der Kammen RA, Clark K, van der Valk M, Michiels F and Collard JG: Mice deficient in the Rac activator Tiam1 are resistant to Ras-induced skin tumours. Nature 417: 867-871, 2002.

14. Adam L, Vadlamudi RK, McCrea P and Kumar R: Tiam1 overexpression potentiates heregulin-induced lymphoid enhancer factor-1/beta-catenin nuclear signaling in breast cancer cells by modulating the intercellular stability. J Biol Chem 276: 28443-28450, 2001.

15. Minard ME, Ellis LM and Gallick GE: Tiam1 regulates cell adhesion, migration and apoptosis in colon tumor cells. Clin Exp Metastasis 23: 301-313, 2006.

16. Engers R, Mueller M, Walter A, Collard JG, Willers R and Gabbert HE: Prognostic relevance of Tiam1 protein expression in prostate carcinomas. Br J Cancer 95: 1081-1086, 2006.

17. Huang J, Ye X, Guan J, Chen B, Li Q, Zheng X, Liu L, Wang S, Ding Y, Ding Y and Chen L: Tiam1 is associated with hepatocellular carcinoma metastasis. Int J Cancer 132: 90-100, 2013.

18. Liu H, Shi G, Liu X, Wu H, Fan Q and Wang X: Overexpression of Tiam1 predicts poor prognosis in patients with esophageal squamous cell carcinoma. Oncol Rep 25: 841-848, 2011.

19. Wang H, Wang H, Makki MS, Wen J, Dai Y, Shi Q, Liu Q, Zhou $X$ and Wang J: Overexpression of $\beta$-catenin and cyclinD1 predicts a poor prognosis in ovarian serous carcinomas. Int J Clin Exp Pathol 7: 264-271, 2013.

20. Cui X, Li L, Yan G, Meng K, Lin Z, Nan Y, Jin G and Li C: High expression of NQO1 is associated with poor prognosis in serous ovarian carcinoma. BMC Cancer 15: 244, 2015.

21. Ma Y, Kong J, Yan G, Ren X, Jin D, Jin T, Lin L and Lin Z: NQO1 overexpression is associated with poor prognosis in squamous cell carcinoma of the uterine cervix. BMC Cancer 14: 414, 2014.

22. Liu S, Li Y, Qi W, Zhao Y, Huang A, Sheng W, Lei B, Lin P, Zhu H, Li W and Shen H: Expression of Tiam1 predicts lymph node metastasis and poor survival of lung adenocarcinoma patients. Diagn Pathol 9: 69, 2014.

23. Sander EE, van Delft S, ten Klooster JP, Reid T, van der Kammen RA, Michiels F and Collard JG: Matrix-dependent Tiam1/Rac signaling in epithelial cells promotes either cell-cell adhesion or cell migration and is regulated by phosphatidylinositol 3-kinase. J Cell Biol 143: 1385-1398, 1998.

24. Sander EE, ten Klooster JP, van Delft S, van der Kammen RA and Collard JG: Rac downregulates Rho activity: Reciprocal balance between both GTPases determines cellular morphology and migratory behavior. J Cell Biol 147: 1009-1022, 1999.

25. Boissier P and Huynh-Do U: The guanine nucleotide exchange factor Tiam1: A Janus-faced molecule in cellular signaling. Cell Signal 26: 483-491, 2014.

26. Yu LN, Zhang QL, Li X, Hua X, Cui YM, Zhang NJ, Liao WT and Ding YQ: Tiam1 transgenic mice display increased tumor invasive and metastatic potential of colorectal cancer after 1,2-dimethylhydrazine treatment. PLoS One 8: e73077, 2013.

27. Li J, Liang S, Jin H, Xu C, Ma D and Lu X: Tiam1, negatively regulated by miR-22, $\mathrm{miR}-183$ and miR-31, is involved in migration, invasion and viability of ovarian cancer cells. Oncol Rep 27: 1835-1842, 2012.

28. Kurman RJ and Shih leM: Pathogenesis of ovarian cancer: Lessons from morphology and molecular biology and their clinical implications. Int J Gynecol Pathol 27: 151-160, 2008.

29. Yang W, Lv S, Liu X, Liu H, Yang W and Hu F: Up-regulation of Tiam1 and Rac1 correlates with poor prognosis in hepatocellular carcinoma. Jpn J Clin Oncol 40: 1053-1059, 2010. 